\title{
Internação por queda em idosos residentes em Florianópolis, em Santa Catarina e no Brasil: tendência temporal 2006-2014
}

\section{Hospitalizations due to accidental falls in elderly living in Florianópolis, Santa Catarina, and Brazil: time trends 2006-2014}

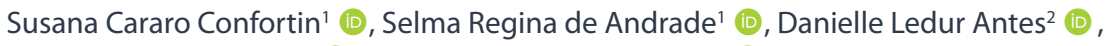 \\ Larissa Pruner Marques² (D), lone Jayce Ceola Schneider ${ }^{3}$ (D) \\ ${ }^{1}$ Programa de Pós-graduação em Enfermagem, Universidade Federal de Santa Catarina (UFSC) - Florianópolis (SC), Brasil. \\ ${ }^{2}$ Programa de Pós-graduação em Saúde Coletiva, Universidade Federal de Santa Catarina (UFSC) - Florianópolis (SC), Brasil. \\ ${ }^{3}$ Universidade Federal de Santa Catarina (UFSC) - Araranguá (SC), Brasil.
}

\begin{abstract}
Como citar: Confortin SC, Andrade SR, Antes DL, Marques LP, Schneider IJC. Internação por queda em idosos residentes em Florianópolis, em Santa Catarina e no Brasil: tendência temporal 2006 a 2014. Cad Saúde Colet, 2020;28(2):251-259. https://doi.org/10.1590/1414-462X202028020255
\end{abstract}

\begin{abstract}
Resumo
Introdução: A queda pode causar graves problemas para os indivíduos, incluindo redução das atividades de vida diária e aumento do medo de andar e de sofrer outra queda, o que pode levar à hospitalização. Objetivo: Analisar a tendência temporal de internações por quedas em idosos em Florianópolis, em Santa Catarina e no Brasil, no período de 2006 a 2014. Método: Estudo de série temporal, com registros de internações por queda, oriundos do Sistema de Informações Hospitalares do Sistema Único de Saúde, em idosos residentes em Florianópolis, em Santa Catarina e no Brasil. Foi utilizado o programa Joinpoint para análise das tendências. Resultados: Foram registradas 703.791 internações por quedas no Brasil, das quais 5\% ocorreram em Santa Catarina e 0,3\% em Florianópolis. Houve tendência crescente no Brasil, de 2008 a 2014, e em Santa Catarina, de 2006 a 2014. Em Florianópolis, ocorreu aumento nas taxas, porém não significativo. Quando analisados os grupos etários, os idosos de 60 a 69 anos foram os que apresentaram as maiores taxas de internação. Conclusão: Foi constatada tendência crescente de internação por quedas em idosos em Santa Catarina (2006-2014) e no Brasil (2008-2014). Idosos de 60 a 69 anos apresentaram as maiores taxas nos três territórios.
\end{abstract}

Palavras-chave: idoso; acidentes por quedas; hospitalização; estudos de séries temporais.

\begin{abstract}
Background: The fall can cause serious problems for individuals, including reduced activities of daily living, increased fear of walking, and suffering another fall, which can have an impact on hospitalization. Objective: To analyze the temporal trend of hospitalizations due to falls in the elderly, in Florianópolis, Santa Catarina, and Brazil, from 2006 to 2014. Method: This is a time-series study with records of hospitalizations from the Hospital Information System of the Unified Health System, due to falls in the elderly of Florianópolis, Santa Catarina, and Brazil. Joinpoint program was used to analyze trends. Results: A total of 703,791 hospitalizations were due to falls in Brazil, of which 5.0\% occurred in Santa Catarina and $0.3 \%$ in Florianópolis. There was a growing trend in Brazil, from 2008 to 2014, and in Santa Catarina, from 2006 to 2014. In Florianópolis, there was an increase in rates, however, it was not significant. When the age groups were analyzed, the group from 60 to 69 years old was the one with the highest admission rates. Conclusion: There was a growing trend of hospitalization for falls in the elderly in Santa Catarina (2006-2014) and Brazil (2008-2014). People aged 60 to 69 years old had the highest rates in the three territories.
\end{abstract}

Keywords: aged; accidental falls; hospitalization; time series studies.

Trabalho realizado na Universidade Federal de Santa Catarina (UFSC) - Florianópolis (SC), Brasil.

Correspondência: Susana Cararo Confortin. E-mail: susanaconfortin@gmail.com

Fonte de financiamento: Coordenação de Aperfeiçoamento de Pessoal de Nível Superior - Brasil (CAPES) - Código de Financiamento 001.

Conflito de interesses: Nada a declarar.

Recebido em: Maio 29, 2018. Aceito em: Jul. 23, 2019
Este é um artigo publicado em sob a licença Creative Commons Attribution, que permite uso, distribuição e reprodução em qualquer meio, sem restrições desde que o trabalho original seja corretamente citado. 


\section{INTRODUÇÃO}

As quedas são consideradas um problema de saúde pública por causa da sua alta morbimortalidade e são definidas como alteração não prevista e não proposital no posicionamento, em que o indivíduo tende a permanecer em lugar inferior a outro, por exemplo, sob a mobília ou no solo/chão, fato não resultante de paralisia súbita, ataque epilético ou força externa' . As quedas estão entre os danos que mais prejudicam os idosos ${ }^{2}$. A prevalência delas nesse grupo etário, em estudos conduzidos no Brasil, varia de $28 \%$ a $37,5 \%{ }^{3-7}$. Em Florianópolis, estudo populacional, de base domiciliar, conduzido com idosos, apresentou prevalência de quedas de $18,2 \%^{8}$.

As quedas podem causar graves problemas para os indivíduos, tais como fraturas, danos de tecidos moles, equimoses, luxações, esfolados e abrasões, lesões musculares e neurológica ${ }^{9}$, perda da capacidade de realizar as atividades de vida diária ${ }^{10}$, aumento do medo de andar e de sofrer outra queda, o que pode levar à hospitalização e, nas ocorrências mais graves, pode culminar em morte do indivíduo ${ }^{10}$. Ainda, são consideradas uma das principais causas de internações de indivíduos com 60 anos ou mais ${ }^{11}$ no Brasil. Segundo dados do IBGE, 30\% da população idosa apresenta problemas em decorrência de quedas no Brasil.

O contingente de indivíduos com 60 anos ou mais no Brasil atingiu mais de 26 milhões de pessoas em 2017, o que correspondia a $12,5 \%$ da população total do país ( $7 \%$ de mulheres e $5,6 \%$ de homens $)^{12}$. Esse fato demonstra que os idosos são o grupo que mais cresce em proporção na pirâmide etária. É observado que o estado de Santa Catarina tem a maior esperança de vida ao nascer, e Florianópolis é considerada uma das capitais mais longevas do país ${ }^{13}$.

Em consonância com o crescimento desse grupo etário e o maior risco de quedas, são consideráveis as implicações econômicas como o impacto para a família, a comunidade e a sociedade. É preciso destacar o aumento dos gastos com as internações por quedas para os serviços de saúde, uma vez que, no ano de 2006, o gasto total foi de mais de R\$ 66 milhões, enquanto, em 2014, mais que duplicou, superando R\$ 142 milhões $^{14}$. Entre o grupo de causas externas, as quedas retratam o maior incremento proporcional de risco de internação, sendo os idosos os mais susceptíveis a esse tipo de agravo ${ }^{15}$.

Por conseguinte, a análise da situação de saúde, especificamente quanto à queda em idosos, é imprescindível, visto que a informação em saúde é um instrumento de apoio decisório, com base no conhecimento da realidade epidemiológica, socioeconômica e demográfica. A vigilância dessa condição pode criar medidas que auxiliem na redução das quedas, contribuir para a diminuição dos custos com internações dessa natureza e reduzir as morbidades e os óbitos consequentes ${ }^{16}$. Assim, o objetivo deste artigo é analisar a tendência de internações por quedas em idosos em Florianópolis, no estado de Santa Catarina e no Brasil, no período de 2006 a 2014.

\section{MÉTODO}

Foi desenvolvido um estudo ecológico de séries temporais que compreendeu dados do período de 2005 a 2015, com dados de internações por quedas em idosos disponíveis no Sistema de Informações Hospitalares do Sistema Único de Saúde (SIHSUS). O SIHSUS tem a finalidade de registrar todas as internações hospitalares e gerar os pagamentos aos estabelecimentos que realizaram esses procedimentos pelo SUS ${ }^{17}$, além de ser um dos sistemas de informação do Departamento de Estatística do SUS, o DATASUS.

Segundo o censo demográfico do Instituto Brasileiro de Geografia e Estatística (IBGE), a população de idosos no Brasil, em 2010, correspondia a 7,2\%. A região Sul, na qual o estado de Santa Catarina está localizado, era uma das mais envelhecidas do país, com 10,5\% dos 6.248.436 habitantes como idosos. Já Florianópolis, a capital do estado de Santa Catarina, apresentava $11,5 \%$ dos habitantes com 60 anos ou mais ${ }^{18}$.

Foram selecionadas as internações disponibilizadas pelo SIHSUS para os residentes da cidade de Florianópolis, do estado de Santa Catarina e do Brasil, no período de 2005 a 2015. Foram incluídas as internações por quedas em idosos (60 anos ou mais), por grupo etário (60-69, 70-79 e 80 anos ou mais), que tiveram como descrição de causa básica a queda, de acordo com o capítulo XX (Códigos W00 a W15 e W17 a W19 da 10a Revisão da Classificação Internacional de Doenças e Problemas Relacionados à Saúde - CID-10), para ambos os sexos. Foram excluídos casos com dados ignorados de sexo e idade. 
As informações referentes à população de idosos (60 anos ou mais) também estão disponíveis nas informações demográficas e socioeconômicas do DATASUS, que utiliza como fonte o IBGE. Para o ano de 2010, foram utilizados os dados do censo demográfico, e para os demais anos, as estimativas populacionais.

Primeiramente, foram calculadas as taxas de internações por causa selecionada, as quedas, seguidas das taxas de internações específicas por idade e das taxas de internações padronizadas pelo método direto, sendo utilizado como padrão a população mundial ${ }^{19}$.

As taxas específicas por idade e as padronizadas calculadas foram empregadas na análise da tendência de internações por meio da estimativa de modelos de regressão linear segmentada. Por causa do pequeno número de casos em determinados estratos, foi utilizada a média móvel centrada em três termos para suavizar a série histórica ${ }^{20}$. Desta forma, as tendências serão apresentadas no período de 2006 a 2014.

Para a estimativa da tendência de internações e suas modificações, foi utilizado o programa Joinpoint, versão 4.5.0.1 (Statistical Research and Applications Branch, National Cancer Institute, Estados Unidos). O programa realiza uma regressão linear segmentada (joinpoint regression) para estimar a variação anual percentual e identificar pontos em que há modificação da tendência. $O$ modelo escolhido é aquele com o maior número de pontos, em que se manteve a significância estatística $(p<0,05)^{21}$.

A partir do coeficiente de regressão são calculadas a variação anual percentual (APC) e sua significância estatística, estimada pelo método dos mínimos quadrados por um modelo linear generalizado. Para cada segmento de reta, com inclinação estimada, foram calculados os limites do intervalo de $95 \%$ de confiança (IC95\%) ${ }^{21}$.

Embora seguindo rigor metodológico, este estudo dispensou apreciação por comitê de ética, visto que se trata de análise de dados agregados, de domínio público, sem a possiblidade de identificação dos indivíduos.

\section{RESULTADOS}

No período de 2006 a 2014, foram registradas 703.791 internações por causa de quedas no Brasil, que representaram 2,85\% em todo o país. Dessas internações, $5 \%$ ocorreram no estado de Santa Catarina, com um total de 34.019. Já em Florianópolis, houve 1.768 internações por quedas, representando $5 \%$ das internações do estado e 0,3\% do país. A Tabela 1 descreve o número de internações por quedas, a taxa bruta e a taxa ajustada para cada ano no Brasil, no estado de Santa Catarina e no município de Florianópolis.

Tabela 1. Taxas de internações por quedas em idosos* (por 100.000 habitantes) no Brasil, no estado de Santa Catarina e no município de Florianópolis. Florianópolis, SC, Brasil, 2017

\begin{tabular}{|c|c|c|c|c|c|c|c|c|c|}
\hline \multirow[b]{2}{*}{ Ano } & \multicolumn{3}{|c|}{ Brasil } & \multicolumn{3}{|c|}{ Santa Catarina } & \multicolumn{3}{|c|}{ Florianópolis } \\
\hline & 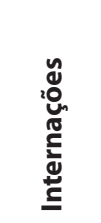 & 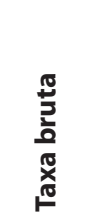 & 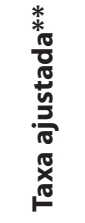 & 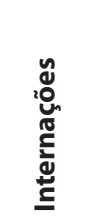 & 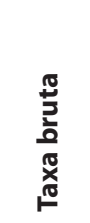 & 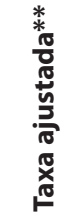 & 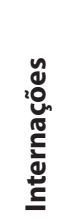 & 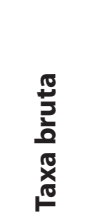 & 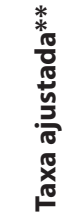 \\
\hline 2006 & 63.509 & 402,7 & 374,0 & 2.904 & 612,1 & 583,3 & 161 & 728,5 & 678,9 \\
\hline 2007 & 67.197 & 369,1 & 338,7 & 3.060 & 527,4 & 494,9 & 142 & 634,9 & 582,8 \\
\hline 2008 & 61.220 & 326,3 & 298,8 & 3.158 & 522,0 & 490,3 & 150 & 520,5 & 472,6 \\
\hline 2009 & 68.566 & 352,9 & 322,9 & 3.432 & 541,7 & 514,0 & 208 & 701,2 & 650,4 \\
\hline 2010 & 79.830 & 387,7 & 353,7 & 3.759 & 572,2 & 539,7 & 224 & 737,9 & 667,8 \\
\hline 2011 & 85.398 & 411,7 & 375,6 & 4.161 & 627,0 & 593,4 & 194 & 624,4 & 598,4 \\
\hline 2012 & 87.369 & 418,2 & 381,9 & 4.225 & 630,6 & 598,9 & 176 & 553,8 & 525,8 \\
\hline 2013 & 93.479 & 423,4 & 388,4 & 4.485 & 598,6 & 565,1 & 226 & 676,6 & 635,8 \\
\hline 2014 & 97.223 & 422,9 & 387,7 & 4.835 & 612,8 & 580,1 & 287 & 838,6 & 797,4 \\
\hline
\end{tabular}

* Os coeficientes dos anos representam a média de um ano anterior, o próprio ano e um ano posterior. ${ }^{* * T a x a}$ ajustada: taxas de internações padronizadas pelo método direto, utilizando como padrão a população mundial. 
As Figuras 1-3 apresentam graficamente a tendência das taxas ajustadas total e segundo a faixa etária ( 60 a 69 anos, 70 a 79 anos e 80 anos ou mais) no Brasil, em Santa Catarina e em Florianópolis, respectivamente. Já a Tabela 2 descreve as variações percentuais que ocorreram no período.

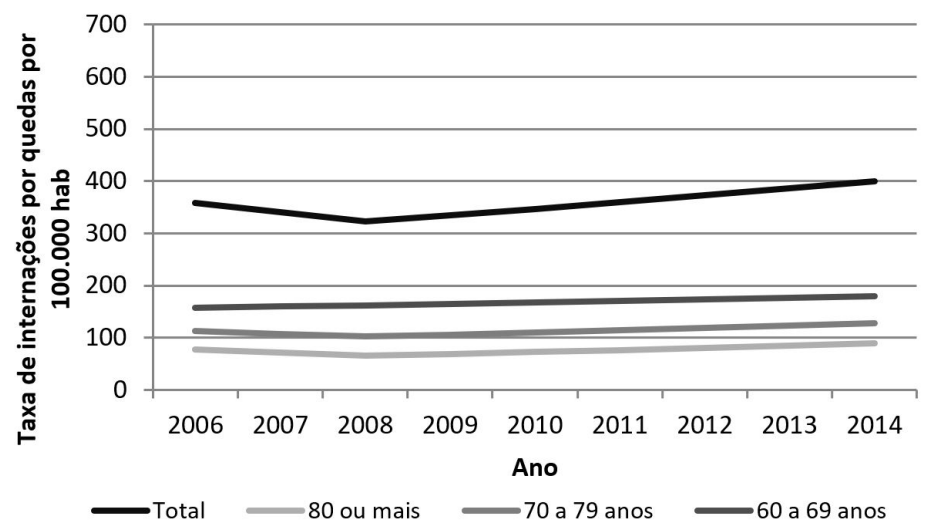

Figura 1. Tendência de internações por quedas em idosos segundo o grupo etário no Brasil, 2006-2014. Florianópolis, SC, 2017.

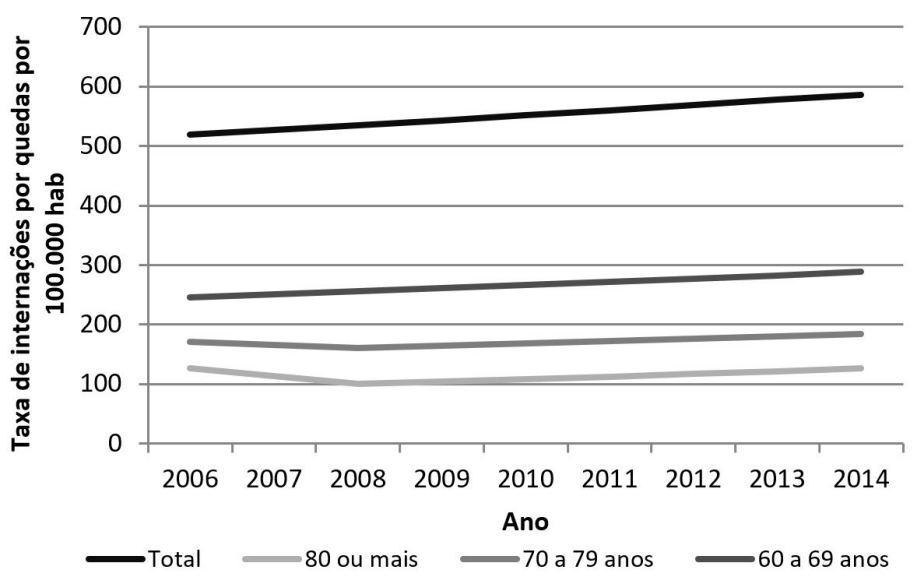

Figura 2. Tendência de internações por quedas em idosos segundo o grupo etário no estado de Santa Catarina, 2006-2014. Florianópolis, SC, 2017.

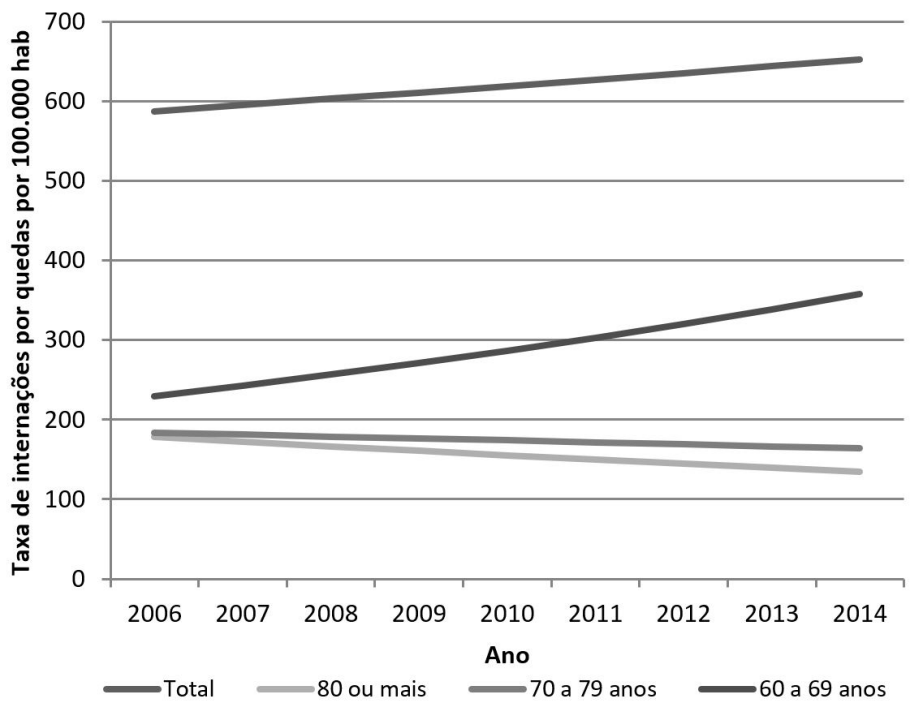

Figura 3. Tendência de internações por quedas em idosos segundo o grupo etário em Florianópolis, 2006-2014. Florianópolis, SC, 2017. 
Tabela 2. Distribuição da variação anual percentual da taxa de internação por quedas, geral e por grupo etário no Brasil, no estado de Santa Catarina e no município de Florianópolis, no período de 2006 a 2014. Florianópolis, SC, 2017

\begin{tabular}{|c|c|c|c|c|c|}
\hline \multicolumn{2}{|c|}{ Brasil } & \multicolumn{2}{|c|}{ Santa Catarina } & \multicolumn{2}{|c|}{ Florianópolis } \\
\hline Período & Variação & Período & Variação & Período & Variação \\
\hline \multicolumn{6}{|l|}{ Geral } \\
\hline 2006-2014 & $1,4(-0,9 ; 3,7)$ & $2006-2014$ & $1,5 \wedge(0,2 ; 2,9)$ & 2006-2014 & $1,3(-0,6 ; 3,3)$ \\
\hline $2006-2008$ & $-5,1(-15,4 ; 6,4)$ & & & & \\
\hline $2008-2014$ & $3,7 \wedge(1,7 ; 5,7)$ & & & & \\
\hline \multicolumn{6}{|l|}{80 anos ou mais } \\
\hline 2006-2014 & $1,6(-0,6 ; 3,9)$ & $2006-2014$ & $0(-3,5 ; 3,5)$ & $2006-2014$ & $-3,5^{\wedge}(-5,7 ;-1,2)$ \\
\hline $2006-2008$ & $-8,2(-18,0 ; 2,8)$ & 2006-2008 & $-10,8(-25,3 ; 6,4)$ & & \\
\hline 2008-2014 & $5,2 \wedge(3,2 ; 7,2)$ & $2008-2014$ & $3,8 \wedge(0,7 ; 7,1)$ & & \\
\hline \multicolumn{6}{|l|}{70 a 79 anos } \\
\hline $2006-2014$ & $1,5(-0,3 ; 3,3)$ & 2006-2014 & $0,9(-0,4 ; 2,2)$ & 2006-2014 & $-1,4(-4,2 ; 1,5)$ \\
\hline $2006-2008$ & $-5,0(-13,1 ; 3,7)$ & 2006-2008 & $-2,9(-9,0 ; 3,6)$ & & \\
\hline $2008-2014$ & $3,8 \wedge(2,3 ; 5,3)$ & 2008-2014 & $2,2 \wedge(1,1 ; 3,3)$ & & \\
\hline \multicolumn{6}{|l|}{60 a 69 anos } \\
\hline 2006-2014 & $1,7 \wedge(0,3 ; 3)$ & 2006-2014 & $2,0 \wedge(0,8 ; 3,3)$ & $2006-2014$ & $5,7 \wedge(3,9 ; 7,5)$ \\
\hline
\end{tabular}

$\wedge$ Valor de $p<0,05$.

No Brasil, houve aumento não significativo da taxa de internação de 1,4\% ao ano. Entretanto, no período de 2008 a 2014, ocorreu aumento significativo anual de 3,7\%. A maior taxa de internação por quedas ocorreu entre aqueles de 60 a 69 anos, com aumento significativo de $1,7 \%$ ao ano. As faixas etárias de 70 a 79 anos e de 80 anos ou mais apresentaram aumento significativo somente no período de 2008 a 2014 (Figura 1 e Tabela 2).

Em Santa Catarina, houve aumento significativo na taxa de internação por quedas em idosos de 1,5\% ao ano no período de 2006 a 2014. Assim como no Brasil, os que apresentaram maior taxa de internação em razão de quedas foram os idosos de 60 a 69 anos, com aumento significativo de $2 \%$ ao ano, sem variações no período. Entre os idosos de 70 a 79 anos e de 80 anos ou mais, houve variação significativa somente no período de 2008 a 2014 (Figura 2 e Tabela 2).

Florianópolis teve aumento da taxa de internação por quedas, entretanto não significativo de 1,3\% ao ano. A faixa etária de 60 anos obteve a maior taxa de internação por quedas, com aumento significativo anual de $5,7 \%$. Já as faixas etárias de 70 a 79 anos e 80 anos ou mais apresentaram redução da taxa de internação por quedas, sendo esta última com queda de 3,5\% ao ano (Figura 3 e Tabela 2).

\section{DISCUSSÃO}

A análise da tendência temporal revelou aumento significativo da variação das taxas de internação por quedas no Brasil, de 2008 a 2014, e em Santa Catarina, de 2006 a 2014. Ao analisar as faixas etárias, as populações de 80 anos ou mais e de 70 a 79 anos, em âmbito nacional e estadual (Santa Catarina), mantiveram a tendência de aumento no período de 2008 a 2014. Quanto maior a faixa etária, maior foi a variação da taxa ao ano, com exceção de Florianópolis.

Considerando o aumento do número de idosos, há maior probabilidade de ocorrência de quedas, uma vez que elas estão ligadas exponencialmente com as mudanças biológicas 
associadas à idade. Um significativo ${ }^{22,23}$ número de idosos deverá desencadear aumento expressivo de quedas, assim como os ferimentos a elas relacionados ${ }^{24}$.

A importância atribuída às quedas, após a publicação da Portaria no 3.213/GM, de 20 dezembro de 2007, sobre a prevenção e o cuidado à osteoporose e quedas, apesar de o principal intuito ser o de alerta à população idosa, pode auxiliar na identificação e nos registros de casos. Conquistas como essa aumentam a discussão sobre o tema entre os profissionais de saúde, além de alertar os idosos quanto à seriedade e às consequências desse problema, o que pode resultar no aumento pela procura dos serviços de saúde. Ainda assim, a sensibilização para tal ocorrência pode refletir na melhora da qualidade dos dados preenchidos nas autorizações de internações hospitalares, possibilitando o uso dos dados em mais estudos ${ }^{25}$.

Ao considerar um panorama geral de fatores, é possível citar também a urbanização no Brasil: em 1991, 75,6\% da população vivia em áreas urbanas; em 2010, a proporção aumentou para $84,4 \%{ }^{26}$. O processo de êxodo, aliada à acelerada transição demográfica, exige a organização e o preparo das cidades, pois não há estrutura do ambiente construído para a mobilidade, como falta de iluminação, calçadas irregulares, cruzamentos perigosos, falta de segurança e de bancos, contribuindo, assim, para a ocorrência de quedas na população idosa ${ }^{27-29}$.

Merece destaque o crescente conjunto de ações e decisões em nível mundial e nacional voltadas à formulação de políticas públicas que enfatizam a prevenção de quedas como uma prioridade de investigação e intervenção. No Brasil, devem ser citados a Política Nacional do Idoso (1994), a Política Nacional de Redução da Morbimortalidade por Acidentes e Violência (2001), o Estatuto do Idoso (2003), a Política Nacional de Saúde da Pessoa Idosa (2006), o Pacto pela Saúde (2006), a Caderneta de Saúde da Pessoa Idosa (2006) e o Caderno de Atenção Básica de Envelhecimento e Saúde da Pessoa Idosa (2006).

Em nível local, devem ser destacados os programas como de apoio domiciliar e de saúde do idoso, intitulado Capital Idoso, além dos protocolos municipais. Outro importante fator a contribuir com o cenário se refere ao aumento da cobertura da Estratégia Saúde da Família, que promove maior acesso aos serviços de saúde, esclarecimento sobre os riscos de quedas, estratégias de prevenção e ações de promoção da saúde ${ }^{30}$. Apesar desses esforços, ainda há aumento das taxas de internação por quedas.

Em Florianópolis, a redução da variação das taxas entre os idosos de 80 anos ou mais pode estar atrelada às condições socioeconômicas. O município destaca-se pelo alto índice de desenvolvimento humano municipal (IDH-M) e de longevidade, além de alta expectativa de vida, comparada à média nacional ${ }^{26}$. Em 2010, a renda per capita foi de $\mathrm{R} \$ 1.374,96$, a maior entre as regiões metropolitanas (RM), e o Índice de Gini foi de 0,52 , o segundo menor entre as RM, o que demonstra um perfil populacional de maior renda e menor desigualdade em sua distribuição.

A região Sul destaca-se pela menor média de permanência hospitalar por quedas, no Brasil $^{31}$. As condições econômicas favoráveis, que acabam aumentando as possibilidade de estruturação de uma rede de cuidados domiciliares, podem reduzir o número de dias de internação do idoso, o que diminui o risco de quedas do leito ${ }^{24}$. Tais condições podem também proporcionar adaptações no ambiente residencial, dispor de mais oportunidades para manter-se ativo e saudável, assim como residir em um bairro com melhor estrutura referente ao ambiente construído ${ }^{32}$.

Apesar de não ter sido investigada no presente estudo, a taxa de mortalidade por quedas, ao comparar os dados de internações por quedas, permite observar que, apesar de as internações terem sido maiores em idosos jovens, as taxas de mortalidade aumentaram com a idade, principalmente em Florianópolis ${ }^{33}$. Com isso, pode ser constatado que idosos com idades mais elevadas tendem a ter eventos de quedas mais fatais e, portanto, não chegam a ser internados, o que pode ser explicado justamente pela maior fragilidade que acomete os idosos mais longevos ${ }^{34}$.

Apesar do contingente populacional maior na faixa etária de 60 a 69 anos $^{35}$, geralmente as condições físicas como cognição e funcionalidade estão preservadas, justificando, assim, as variações menores na taxa de internações por quedas, comparada a outras idades. Contudo, Florianópolis apresenta um cenário diferente, com maior variação observada entre os mais jovens. 
De acordo o IBGE ${ }^{36}$, a população idosa concentra-se nos grandes centros urbanos, nas capitais dos estados, onde vivem $25 \%$ da população idosa do país, o que aumenta a probabilidade do evento. Mascarenhas e Barros ${ }^{25}$, ao investigarem a internação por causas externas, encontraram maior incremento no coeficiente de internação hospitalar no Sul do Brasil e explicaram esse resultando a partir da estrutura etária da população, mencionando que, apesar de a população idosa apresentar crescimento proporcional em todo o país, é na região Sul onde se encontra a maior participação de idosos sobre o total de residentes, o que pode também justificar os resultados da presente investigação para a cidade de Florianópolis.

$\mathrm{O}$ uso de base de dados secundárias para investigações permite a obtenção rápida e menos custosa das informações, possuindo amplitude geográfica e populacional, além de temporal. Esses são aspectos que possibilitam a análise da situação em saúde e oferecem subsídios para uma gestão baseada em evidências. No entanto, assim como toda investigação que utiliza base de dados secundárias, o presente estudo apresenta limitação quanto à qualidade, confiabilidade e validade dos dados do SIHSUS. Com relação ao preenchimento, as informações sobre diagnóstico secundário nesse sistema são as que apresentam maior subnotificação $0^{37}$, o que não se aplica ao presente estudo. Já as informações não preenchidas, como sexo e idade, e as diferenças regionais no preenchimento das AlH podem subestimar o número de internações identificadas por quedas. Ainda assim, foi observado o aumento das taxas ao longo do período.

Foi constatada uma tendência temporal crescente do número de internações por quedas no Brasil, de 2008 a 2014, e em Santa Catarina, de 2006 a 2014. Quando analisados os grupos etários, os idosos de 60 a 69 anos foram os que apresentaram as maiores taxas no Brasil e em Santa Catarina, com destaque para Florianópolis. Apesar das altas taxas de internações por quedas, em Florianópolis, no período de 2006 a 2014, não foi identificada variação no percentual anual dos idosos de 70 a 79 anos; porém, os idosos com 80 anos ou mais apresentaram redução da variação anual.

Ao considerar as diversas consequências que a queda pode gerar tanto no âmbito individual, com o surgimento de incapacidades, morbidades, risco de mortalidade e institucionalização, como no coletivo, com os custos hospitalares, a vigilância e a intervenção nesse panorama em saúde, a vigilância permanente deve ser mantida e enfatizada. Em um período em que os recursos financeiros estão escassos, a avaliação do impacto é fundamental. Em 2014, somente as internações por quedas entre os idosos representou um custo de R\$ 142.029.461,67, sendo quase $9 \%$ de todo o custo hospitalar no SUS do Brasil ${ }^{14}$.

Medidas e ações adequadas e viáveis para melhora e manutenção da qualidade de vida dessa população podem reduzir as taxas por queda, visto ser uma causa externa evitável. As ações devem ser multifatoriai ${ }^{32,38}$, sobretudo a importância de adaptação de ambientes construídos, a fim de favorecer envelhecimento ativo e saudável. Em nível residencial, as modificações devem visar ao aumento da segurança, além de estimular o controle e a autonomia e facilitar a interação social. Ainda, é preciso haver suplementação de vitamina D para idosos com deficiência, bem como uma avaliação da acuidade visual, da interação medicamentosa e polifarmácia. Todos esses cuidados, aliados a programas de exercícios físicos que estimulem principalmente o desenvolvimento e a manutenção da força, equilíbrio e marcha, revelam-se a estratégia mais eficiente na prevenção das quedas ${ }^{38,39}$.

Diante do cenário exposto pelo presente trabalho, há grande impacto também na atuação dos profissionais de saúde, os quais precisam estar preparados para atender aos desafios do envelhecimento populacional, bem como para orientar e acompanhar os cuidados proporcionados pela família e cuidadores, considerando que resultados de uma revisão integrativa evidenciam que poucas das estratégias recomendas na literatura para a prevenção de quedas são adotadas pelos idosos ${ }^{40}$.

O aumento do número de idosos traz novos desafios e a necessidade de buscar soluções para os problemas de saúde dessa população, além de ser imprescindível propor medidas de ações adequadas e viáveis para a melhora e a manutenção da qualidade de vida desses idosos. Ao considerar o impacto das quedas na vida dos idosos, é fundamental a priorização de ações para reduzi-las por meio da prevenção e promoção de um envelhecimento ativo. 


\section{AGRADECIMENTOS}

O presente trabalho foi realizado com apoio da Coordenação de Aperfeiçoamento de Pessoal de Nível Superior - Brasil (CAPES) - Código de Financiamento 001.

\section{REFERÊNCIAS}

1. Kellogg International Work Group on the Prevention of Falls by the Elderly. The prevention of falls in later life. A report of the Kellogg International Work Group on the Prevention of Falls by the Elderly. Dan Med Bull. 1987;34(4 Suppl):1-24. PMid:3595217.

2. Meschial WC, Soares DFPP, Oliveira NLB, Nespollo AM, Silva WA, Santil FLP. Elderly victims of falls seen by pre hospital care: gender differences. Rev Bras Epidemiol. 2014; 17(1):3-16.

3. Vieira LS, Gomes AP, Bierhals IO, Farías-Antúnez S, Ribeiro CG, Miranda VIA et al. Quedas em idosos no Sul do Brasil: prevalência e determinantes. Rev Saúde Pública. 2018;52:22. http://dx.doi.org/10.11606/ s1518-8787.2018052000103.

4. Nascimento JS, Tavares DMS. Prevalência e fatores associados a quedas em idosos. Texto Contexto Enferm. 2016;25(2):e0360015. http://dx.doi.org/10.1590/0104-07072016000360015.

5. Siqueira FV, Facchini LA, Piccini RX, Tomasi E, Thumé E, Silveira DS et al. Prevalência de quedas em idosos e fatores associados. Rev Saúde Pública. 2007;41(5):749-56.

6. Motta LB, Aguiar AC, Coutinho ESF, Huf G. Prevalência e fatores associados a quedas em idosos em um município do Rio de Janeiro. Rev Bras Geriatr Gerontol. 2010;13(1):83-91. .

7. Siqueira FV, Facchini LA, Silveira DS, Piccini RX, Tomasi E, Thumé E et al.. Prevalence of falls in elderly in Brazil: a countrywide analysis. Cad Saúde Pública. 2011;27(9):1819-26. .

8. Confortin SC, Schneider IJC, Antes DL, Cembranel F, Ono LM, Marques LP, et al. Life and health conditions among elderly: results of the EpiFloripa Idoso cohort study. Epidemiol Serv Saude. 2017;26(2):305-17. http://dx.doi.org/10.5123/s1679-49742017000200008.

9. Maia BC, Viana PS, Arantes PMM, Alencar MA. Consequences of falls in older people living in the community. Rev Bras Geriatr Gerontol. 2011;14(2):381-93. http://dx.doi.org/10.1590/S1809-98232011000200017

10. Costa NR, Pinto LF. Avaliação de programa de atenção à saúde: incentivo à oferta de atenção ambulatorial e a experiência da descentralização no Brasil. Ciênc Saúde Coletiva. 2002;7(4):907-23.

11. Ribeiro AP, de Souza ER, Atie S, de Souza AC, Schilithz AO. A influência das quedas na qualidade de vida de idosos. Ciênc Saúde Coletiva. 2008; 13(4):1265-73.

12. Instituto Brasileiro de Geografia e Estatística. Projeção da população do Brasil e das Unidades da Federação [Internet]. Rio de Janeiro: IBGE; 2018 [citado em 2018 out 29]. Disponível em: https://ww2.ibge.gov.br/ apps/populacao/projecao/.

13. Instituto Brasileiro de Geografia e Estatística. Tábua completa de mortalidade para o Brasil - 2015: Breve análise da evolução da mortalidade no Brasil [Internet]. Rio de Janeiro: IBGE; 2016 [citado em 2018 out 29]. Disponível em: ftp://ftp.ibge.gov.br/Tabuas_Completas_de_Mortalidade/Tabuas_Completas_de_ Mortalidade_2015/tabua_de_mortalidade_analise.pdf

14. Brasil. Ministério da Saúde. Sistema de Informações Hospitalares do SUS (SIH/SUS) [Internet]. Brasília, DF: Ministério da Saúde; 2018 [citado em 2018 out 29]. Disponível em: http://tabnet.datasus.gov.br/cgi/ deftohtm.exe?sih/cnv/niuf.def

15. Gawryszewski VP. A importância das quedas no mesmo nível entre idosos no estado de são paulo. Rev Assoc Med Bras. 2010;56(2):162-167. http://dx.doi.org/10.1590/S0104-42302010000200013.

16. Buksman S, Vilela A, Pereira S, Lino V, Santos V. Projeto diretrizes: quedas em idosos: prevenção [Internet]. São Paulo: Associação Médica Brasileira, Conselho Federal de Medicina. Sociedade Brasileira Geriatria e Gerontologia; 2008 [cited 2018 Nov 02]. Disponível em: www.projetodiretrizes.org.br/projeto_ diretrizes/082.pdf

17. Brasil. Ministério da Saúde. Sistema de Informações Hospitalares do SUS [Internet]. Brasília: Ministério da Saúde; 2018 [citado em 2018 out 5]. Disponível em: http://datasus.saude.gov.br/sistemas-e-aplicativos/ hospitalares/sihsus

18. Instituto Brasileiro de Geografia e Estatística. Censo Demográfico 2010. Características da população e dos domicílios: resultados do universo [Internet]. Rio de Janeiro: IBGE; 2000 [cited 2018 out 5]. Disponível em: https://ww2.ibge.gov.br/home/estatistica/populacao/censo2010/

19. Doll R, Payne $P$, Waterhouse J. Cancer incidence in five continents: a technical report. Berlin: SpringerVerlag; 1966. http://dx.doi.org/10.1007/978-3-642-85849-9. 
20. Latorre MRDO, Cardoso MRA. Análise de séries temporais em epidemiologia: uma introdução sobre os aspectos metodológicos. Rev Bras Epidemiol. 2001; 4:145-52. http://dx.doi.org/10.1590/S1415790X2001000300002.

21. Kim HJ, Fay MP, Feuer EJ, Midthune DN. Permutation tests for joinpoint regression with applications to cancer rates. Stat Med. 2000;19(3):335-51. http://dx.doi.org/10.1002/(SICI)1097-0258(20000215)19:3<335::AIDSIM336>3.0.CO;2-Z. PMid:10649300.

22. Nunes A. Os custos do tratamento da saúde dos idosos no Brasil. In: Camarano AA, organization. Muito além dos 60: os novos idosos brasileiros. Rio de Janeiro: IPEA; 1999. p. 345-368.

23. Morosini S, Marques APO, Leal MCC, Marino JG, Melo HMA. Custo e tempo de permanência hospitalar de idosos residentes em Recife - PE. Geriatr Gerontol Aging. 2011;5(2):91-8.

24. World Health Organization. World Health Organization global report on falls prevention in older age [Internet]. Genebra; 2007 [citado em 2018 out 7]. Disponível em: http://www.who.int/ageing/publications/ Falls_prevention7March.pdf

25. Mascarenhas MDM, Barros MBA. Evolução das internações hospitalares por causas externas no sistema público de saúde - Brasil, 2002 a 2011. Epidemiol Serv Saúde. 2015;24(1):19-29. http://dx.doi.org/10.5123/ S1679-49742015000100003

26. Instituto de Pesquisa Econômica Aplicada. Programa das Nações Unidas para o Desenvolvimento no Brasil. Atlas do Desenvolvimento Humano [Internet]. Brasília; 2010 [citado em 2018 out 7]. Disponível em: http://www.atlasbrasil.org.br/2013/pt/consulta/.

27. Küchemann BA. Envelhecimento populacional, cuidado e cidadania: velhos dilemas e novos desafios. Soc Estado. 2012;27(1):165-80. http://dx.doi.org/10.1590/S0102-69922012000100010.

28. Mascarenhas MDM, Barros MBA. Evolução das internações hospitalares por causas externas no sistema público de saúde - Brasil, 2002 a 2011. Epidemiol Serv Saúde. 2015; 24(1):19-29. http://dx.doi.org/10.5123/ S1679-49742015000100003.

29. Pereira GN, Morsch P, Lopes DGC, Trevisan MD, Ribeiro A, Navarro JHN, et al. Fatores socioambientais associados à ocorrência de quedas em idosos. Ciênc Saúde Coletiva. 2013;18:3507-14. . http://dx.doi. org/10.1590/S1413-81232013001200007.

30. Malta DC, Santos MAS, Stopa SR, Vieira JEB, Melo EA, Reis AAC. A Cobertura da Estratégia de Saúde da Família (ESF) no Brasil, segundo a Pesquisa Nacional de Saúde, 2013. Ciênc Saúde Coletiva. 2016; 21:32738. http://dx.doi.org/10.1590/1413-81232015212.23602015

31. Barros IFO, Pereira MB, WeillerTH, Anversa ETR. Internações hospitalares por quedas em idosos brasileiros e os custos correspondentes no âmbito do Sistema Único de Saúde. Kairós Gerontol. 2015;18(4):18.

32. Falsarella GR, Gasparotto LPR, Coimbra AMV. Quedas: conceitos, frequências e aplicações à assistência ao idoso. Rev Bras Geriatr Gerontol. 2014; 17:897-910. http://dx.doi.org/10.1590/1809-9823.2014.13064

33. Antes DL, Schneider IJC, d'Orsi E. Mortalidade por queda em idosos: estudo de série temporal. Rev Bras Geriatr Gerontol. 2015;18(4):769-78.

34. Song X, Mitnitski A, Rockwood K. Prevalence and 10-year outcomes of frailty in older adults in relation to deficit accumulation. J Am Geriatr Soc. 2010;58(4):681-7. http://dx.doi.org/10.1111/j.15325415.2010.02764.x. PMid:20345864.

35. Instituto Brasileiro de Geografia e Estatística. Indicadores sociais municipais: uma análise dos resultados do universo do censo demográfico 2010. Estudos e pesquisas informação demográfica e socioeconômica número 28. [Internet]. Rio de Janeiro: IBGE; 2011 [citado em 2018 Out 26]. Disponível em: https://biblioteca. ibge.gov.br/pt/biblioteca-catalogo?view=detalhes\&id=254598

36. Instituto Brasileiro de Geografia e Estatística. Síntese de Indicadores Sociais: uma análise das condições de vida da população brasileira. [Internet]. Rio de Janeiro: IBGE; 2012 [citado em 2018 Out 26]. Disponível em: https://biblioteca.ibge.gov.br/visualizacao/livros/liv98965.pdf

37. Machado JP, Martins M, Leite IC. Qualidade das bases de dados hospitalares no Brasil: alguns elementos. Rev Bras Epidemiol. 2016;19:567-81. http://dx.doi.org/10.1590/1980-5497201600030008.

38. Terroso M, Rosa N, Torres Marques A, Simoes R. Physical consequences of falls in the elderly: A literature review from 1995 to 2010. Eur Rev Aging Phys Act. 2014;11(1):51-9. http://dx.doi.org/10.1007/s11556013-0134-8.

39. Vieira ER, Palmer RC, Chaves PHM. Prevention of falls in older people living in the community. BMJ. 2016;353:i1419. http://dx.doi.org/10.1136/bmj.i1419. PMid:27125497.

40. Wilkinson A, Meikle N, Law P, Yong HJ, Butler P, Kim J, et al. How older adults and their informal carers prevent falls: An integrative review of the literature. Int J Nurs Stud. 2018;82:13-9. http://dx.doi.org/10.1016/j. ijnurstu.2018.03.002. PMid:29573592. 